\title{
PALINOLOGIA DOS CARVÕES PALEOZÓICOS DO ESTADO DE SÃO PAULO. II - O CARVÃO DE MONTE MOR
}

\author{
Paulo Alves de SOUZA \\ Antônio Roberto SAAD \\ Murilo Rodolfo de LIMA ( $†$ )
}

\begin{abstract}
RESUMO
Este trabalho se constitui na segunda contribuição a partir do desenvolvimento dos estudos palinológicos dos carvões paleozóicos do Estado de São Paulo, que inclui os carvões de Buri, Monte Mor e Cerquilho. Os resultados das análises palinológicas do carvão de Monte Mor são apresentados, com a descrição de vinte e seis táxons, dos quais dois são inéditos para a Bacia do Paraná (Punctatisporites resolutus Playford, 1971 e Apiculiretusispora $\mathrm{cf}$. A. coalescens Menéndez \& Azcuy, 1973). A maioria dos táxons descritos ocorrem, também, em Buri e em Araçoiaba da Serra, permitindo assim a correlação palinológica com as associações ali apresentadas e, conseqüentemente, sua correlação estratigráfica. Uma idade westphaliana é sugerida para o carvão de Monte Mor, assim como já fôra proposto para Buri e Araçoiaba da Serra, referíveis ao intervalo bioestratigráfico informal "Pré-G". Fora do Brasil, a única possibilidade de correlação é com as biozonas westphalianas da Bacia de Paganzo, Argentina. As informações paleoambientais são apresentadas com base na freqüência dos morfogrupos palinológicos considerados e os contextos geológico e estratigráfico são discutidos.
\end{abstract}

\section{ABSTRACT}

This paper is a second contribution to the palynological study of the Palaeozoic coals of the Itararé Subgroup in São Paulo State, in the Northeast Paraná Basin. It comprises a systematic description of twenty-six species of spores and pollen grains from coal and silty-coal samples of Monte Mor, from where only macrofloristic data are well known. Among the taxa studied, three are reported for the first time from the Paraná Basin: Punctatisporites resolutus Playford, 1971 and Apiculiretusispora sp. cf. A. coalescens Menéndez \& Azcuy, 1973. The majority of the taxa studied from Monte Mor has also been reported in the Buri and Araçoiaba da Serra assemblages, suggesting that the Monte Mor deposits are correlative with the latter assemblages, thus placing the three in the informal "Pré-G" interval. Outside Brazil, the sole possibility of palynological correlation is with the Carboniferous palynozones of the Paganzo Basin, Argentina. A Westphalian age is attributed to the Monte Mor coal, as had been suggested for those from Buri and Araçoiaba da Serra. The frequencies of the main morphographic groups are presented in order to give paleoecological information. Some geological and stratigraphic aspects are also discussed.

\section{INTRODUÇÃO}

Os níveis de carvão de Monte Mor, Buri e Cerquilho, presentes no Grupo Tubarão no Estado de São Paulo, são possuidores, junto com os sedimentos associados, de grande quantidade de megafósseis vegetais que, ao longo do tempo, têm sido objeto de inúmeros estudos, cujos resultados os têm posicionado estratigraficamente, do mais velho para o mais novo, na ordem acima apresentada.

Macrofósseis vegetais, megásporos e micrósporos são usados para atribuir idade às camadas portadoras de carvão da região de Monte Mor apresentando, por vezes, resultados contraditórios. O melhor posicionamento estrati-

† Falecido em 27 de março de 1995. 
gráfico destas camadas se faz necessário tendo em vista a potencialidade fossilífera apresentada, as dificuldades de correlação de suas camadas com outras da mesma unidade e sua determinação bioestratigráfica na seqüência neopaleozóica da Bacia. O último trabalho de síntese da bioestratigrafia do Subgrupo Itararé foi realizado por PETRI \& SOUZA (1993), que aceitaram uma idade carbonífera para o carvão de Monte Mor, mais jovem que o carvão de Buri, com base nos dados preliminares divulgados por SOUZA et al. (1993a).

Embora existam dados relativos a palinomorfos registrados em Monte Mor, o nível de abordagem adotado até agora pode ser considerado demasiadamente superficial, dificultando a obtenção de conclusões satisfatórias, o que também ocorre com os carvões da região de Cerquilho. Os níveis de Buri foram recentemente estudados no projeto "Palinologia dos Carvões Paleozóicos do Estado de São Paulo", com auxílio FAPESP (Processo n. 89/0162-0) que envolveu o estudo dos três carvões citados, cujos resultados demonstraram ser o carvão de Buri o mais antigo entre os carvões analisados, contrariando opiniões anteriores (SOUZA et al., 1993b). Desta forma, o melhor detalhamento das informações palinológicas do carvão de Monte Mor se faz importante, principalmente tendo em vista tratar-se de localidade-tipo de algumas associações bioestratigráficas de macrovegetais fósseis (RÖSLER, 1978; MILLAN, 1987).

Este trabalho objetiva ilustrar os palinomorfos verificados no carvão de Monte Mor, bem como discutir seu significado bioestratigráfico e valor no contexto geológico.

\section{CONSIDERAÇÕES GERAIS}

\subsection{Contexto geológico}

O depósito de carvão de Monte Mor, Estado de São Paulo, situa-se no flanco nordeste da Bacia do Paraná, em área de predominância dos sedimentos do Grupo Tubarão, Subgrupo Itararé (FIGURA 1). Nessa porção do Estado, o limite inferior dessa unidade se dá em discordância com o embasamento cristalino précambriano, e o superior com a Formação Tatuí, de idade permiana. Os mergulhos regionais são para oeste, suaves, com valor médio de $1^{\circ}$ (SAAD, 1977). Vários sistemas lineares de falhas e falhas isoladas são cartografadas nessa região, mas nenhuma estrutura em particular foi identificada até então. Em inúmeros locais a seqüência das exposições se encontra prejudicada por coberturas cenozóicas e por intrusões de rochas básicas juro-cretáceas, cujo corpo mais expressivo ocorre na cidade de Campinas.

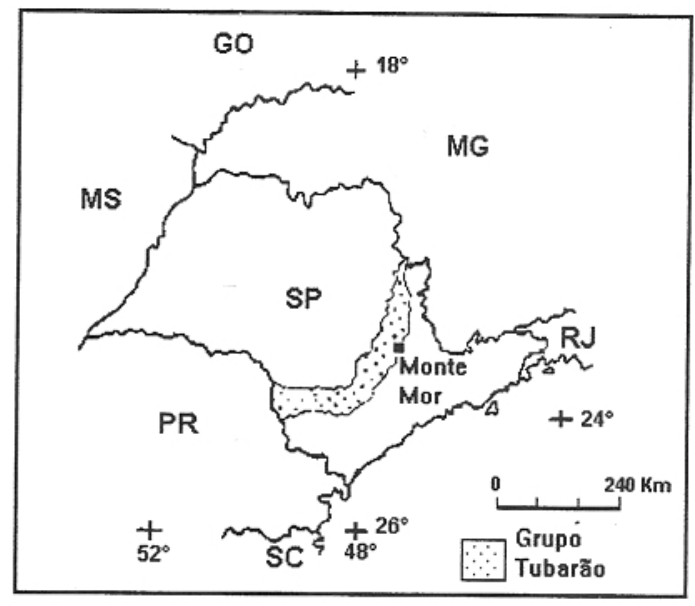

FIGURA 1 - Localização da área onde aflora o carvão de Monte Mor.

De um modo geral, o Subgrupo Itararé aflorante nessa borda da Bacia do Paraná se caracteriza, tanto em superfície como em subsuperficie, por apresentar uma grande diversidade litológica, onde se destacam arenitos de granulometria variada, desde muito finos a conglomeráticos, diamictitos, ritmitos, siltitos, folhelhos, argilitos e carvão (SAAD, 1977; SANTOS, 1979, 1987; SOUZA FILHO, 1986).

SOUZA FILHO (op. cit.) elaborou um mapeamento faciológico do Subgrupo Itararé, na Folha Campinas (SF-23-Y-A-V), com base em sistemas deposicionais, e cujos resultados serão aqui utilizados, visto que, através dessa metodologia moderna de análise estratigráfica, foi possível a reconstrução paleogeográfica da seqüência analisada. Assim, de acordo com esse autor, o mapeamento faciológico permitiu a individualização de um grande número de fácies litológicas, agrupadas em fácies de arenitos, fácies de conglomerados, fácies de diamictitos, fácies de lamitos e fácies de calcáreos. Apesar desses fácies ocorrerem em diversas posições estratigráficas, o seu agrupamento, em associações, permitiu a interpretação de seis sistemas deposicionais principais, cuja evolução originou o trato deposicional hoje preservado sob o nome de Subgrupo Itararé. São eles: sistemas glaciais (2 tipos), sistema de leques subaquosos, sistema plataformal, sistema costeiro, sistema deltaico e, por fim, sistema de leques aluviais.

O carvão de Monte Mor se insere no lobo deltaico homônimo, do Sistema Deltaico da Rodovia do Açúcar, cujo empilhamento sedimentar de fácies pode ser observado na FIGURA 2 (página 87 de SOUZA FILHO, op. cit.). O 
conjunto faciológico detectado, bem como o empilhamento sedimentar reconhecido, refletem uma situação fisiográfica dominada por sistema deltaico, em mares rasos plataformais, onde se registram eventos progradacionais e retrogradacionais. Nesse contexto, a seqüência basal representa o domínio inicial prodeltaico (associação basal de finos com siltitos intercalados por arenitos finos), que passa para as fácies de frente deltaica proximal (arenitos muito finos), planície deltaica/planície aluvial (conglomerados maciços, arenitos com estratificação cruzada tabular e acanalada, lamitos arroxeados e lamitos com carvão, arenitos plano paralelos e com marcas onduladas), seguidos de fácies finas no topo, tais como arenitos com marcas de onda, estratificação cruzada de alto ângulo, siltitos e folhelhos.

Fora da área estudada, podem ser observados outros sistemas deltaicos, como os das regiões de Santa Bárbara e Capivari, como parte do Sistema Deltaico da Rodovia do Açúcar (SOUZA FILHO, op. cit.).

\subsection{Paleontologia}

Com relação à fauna fóssil, restos de peixes paleoniscídeos, pertencentes a pelo menos 2 espécies distintas, uma das quais relacionadas ao gênero Elonichthys, foram registrados por
BARBOSA \& ALMEIDA (1949) em um nível a cerca de $0,5 \mathrm{~m}$ abaixo da camada de carvão de Monte Mor. MILLAN (1975) registrou a ocorrência de conchostráceos cf. Leaia sp. e euripterídeos cf. Eurypterus sp. em níveis associados aos megafósseis vegetais. Mais recentemente, MARTINS NETO et al. (1988) noticiaram a ocorrência de uma impressão de inseto fóssil em sedimento proveniente da localidade Sítio da Mina em Monte Mor, abaixo dos níveis de carvão. Este seria o registro mais antigo de inseto no Subgrupo Itararé, correspondendo a uma nova família da subordem Permotrichoptera, com características primitivas, compatíveis com a idade carbonífera, apresentando diferenças das formas conhecidas da subordem no Permiano.

Contudo, são os macrofósseis vegetais provenientes da capa da camada carbonosa e de folhelhos da região de Monte Mor os elementos mais comumente encontrados no carvão de Monte Mor, já noticiados preliminarmente por BARBOSA \& ALMEIDA (1949) e BARBOSA (1958). Nesse aspecto, o maior número de informações é proveniente dos trabalhos de MILLAN (e.g. 1972; 1975; 1981a; 1981b), com suas implicações bioestratigráficas discutidas em RÖSLER (1978) e MILLAN (1987). Com base nestes trabalhos, a associação de macrofósseis vegetais é aceita como neocarbonífera, pela mis-

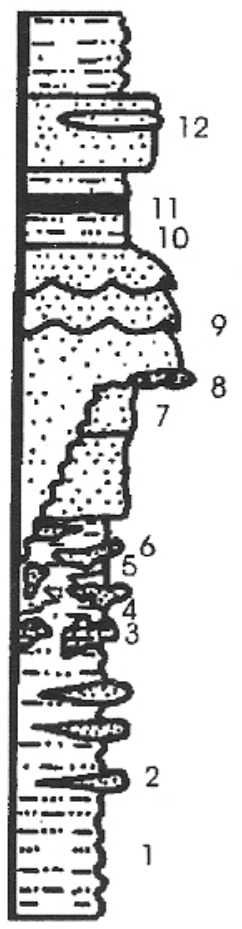
12 arenito fino, médio, bimodal, c/estratificação cruzada de alto ângulo
11 carvão
10 siltito $\mathrm{c} /$ restos vegetais e fósseis de água doce
9 arenito grosso com ciclos de afina- mento até médio e estratificação cruzada
8 conglomerado em depósitos irregulares
7 arenito muito fino a fino, c/estratifi- cação plano-paralela
6 arenito fino c/microestratificação cruzada, deformado
5 lamito arenoso conglomerático maciço
4 siltito laminado deformado
3 calcáreo micrítico com restos fosfáticos deformados
2 arenito muito fino, c/marcas onduladas e microcruzadas
1 siltito cinza maciço ou laminado

\author{
prodelta/ \\ duna litorânea \\ planície de \\ inundação \\ canal \\ praia/frente
deltaica
frente deltaica \\ prodelta/frente \\ deltaica
}

prodelta

FIGURA 2 - Seqüência ideal de fácies do lobo deltaico de Monte Mor (modificado a partir de SOUZA FILHO, 1986) com representação dos litotipos e interpretação litofaciológica. 
tura de Lycopsida e Sphenopsida arborescentes com formas como Nothorhacopteris, Botrychiopsis, Adiantites, Ginkgophyllum e coníferas primitivas (Paranocladus, Buriadia) e ainda pela ausência total das glossopterídeas. Para RÖSLER (1978), a assembléia de vegetais fósseis de Monte Mor caracterizaria a tafoflora mais antiga do Neopaleozóico da Bacia do Paraná, Tafoflora A, com idade posicionada no Stephaniano, proposição aceita por ARCHANGELSKY \& CUNEO (1991), com base em comparações com material do Neopaleozóico argentino.

O único trabalho anterior com relação à palinologia de Monte Mor foi o realizado por DAEMON (1974), que verificou a ocorrência de esporos lisos (Laevigatosporites sp.), esporos apiculados (Verrucosisporites sp.), zonados (Vallatisporites, Hymenozonotriletes sp.), grãos de pólen monossacados (Plicatipollenites gondwanensis, $P$. indicus, $P$. spp., Virkkipollenites cf. $V$. obscurus, $V$. cf. $V$. triangularis, $V$. spp., Potonieisporites spp., Sahnites gondwanensis, $S$. sp., Crucisaccites cf. C. latissulcatus), grãos de pólen estriados (Striomonosaccites spp., Faunipollenites sp., Protohaploxypinus sp.), além de Tasmanites sp.? e hystricosphaerídeos (sic) indeterminados considerados retrabalhados.

A abordagem taxonômica deste estudo foi superficial, com ausência de fotomicrografias que permitam melhores comparações. Contudo, vale ressaltar que DAEMON (1974) posicionou o carvão de Monte Mor no intervalo $\mathrm{H}$, subintervalos $\mathrm{H}_{1} / \mathrm{H}_{2}$, cuja correspondência geocronológica foi atribuída ao Sakmariano.

Como será discutido mais adiante, não apenas as propostas de zoneamento bioestratigráfico e suas correspondências geocronológicas para a Bacia do Paraná sofreram modificações nos últimos anos, em decorrência do avanço dos estudos, mas também o detalhamento sistemático e a melhor correlação das associações no âmbito gondvânico têm sido realizados progressivamente, modificando, profundamente o arcabouço bioestratigráfico da bacia.

\subsection{Materiais e Métodos}

Foram processadas duas amostras: uma amostra de carvão proveniente do rejeito da mina abandonada de Monte Mor, cedida pelo Prof. Dr. Paulo Roberto dos Santos (IG/USP) e pelo segundo autor deste artigo e uma amostra de siltito carbonoso contendo impressões de vegetais fósseis, coletada e cedida pelo Prof. Dr. José H. Millan (Museu Nacional-RJ).
O posicionamento estratigráfico das amostras pode ser visualizado a partir do empilhamento e seqüência ideal de fácies elaborado para a região por SOUZA FILHO (1986), sendo a amostra de siltito carbonoso e o carvão correspondentes às fácies 10 (siltito com restos vegetais e fósseis de água doce) e 11 (carvão), respectivamente.

Os métodos de tratamento empregados para obtenção das lâminas palinológicas foram os habituais em Palinologia, constituindo-se da desagregação física da amostra, por trituração em almofariz e dissolução de seus componentes inorgânicos com HCI e HF e oxidação com $\mathrm{HNO}_{3}$ e solução de Schultze.

Foram confeccionadas 4 lâminas'de cada uma das amostras, as quais se encontram depositadas na Palinoteca do acervo paleontológico do Instituto Geológico/SMA, sob números IG-P-01 a 04 (amostra de carvão) e IGP-05 a 08 (siltito carbonoso). As análises palinológicas e as fotomicrografias foram realizadas em microscópio óptico Carl Zeiss-Jenaval e Axiophot, respectivamente. As coordenadas de localização das lâminas são referentes ao primeiro aparelho, locado no Laboratório de Microscopia do IG/SMA.

\section{SISTEMÁTICA PALINOLÓGICA}

São apresentadas 26 espécies distintas de esporomorfos, incluindo 17 de esporos, 6 de grãos de pólen monossacados e 3 de grãos de pólen estriados, relativas a 19 gêneros, conforme relação sistemática e descrições que seguem.

No posicionamento supragenérico dos táxons verificados foi utilizado o esquema clássico de POTONIÉ (1970) e agrupamentos conforme comumente apresentado na palinologia. São descritos apenas os táxons cuja determinação em nível de espécie esteja aberta sendo, em alguns casos, colocadas algumas observações, quando julgadas necessárias.

\section{Anteturma PROXIMEGERMINANTES Potonié, 1970}

Turma TRILETES (Reinsh) Dettman, 1963

Suprasubturma ACAVATITRILETES (Lüber)

Dettman, 1963

Subturma AZONOTRILETES (Lüber) Dettman, 1963

Infraturma LAEVIGATI (Bennié \& Kidston) Potonié, 1956

Gênero Punctatisporites (Ibrahim) Potonié \& Kremp, 1954 


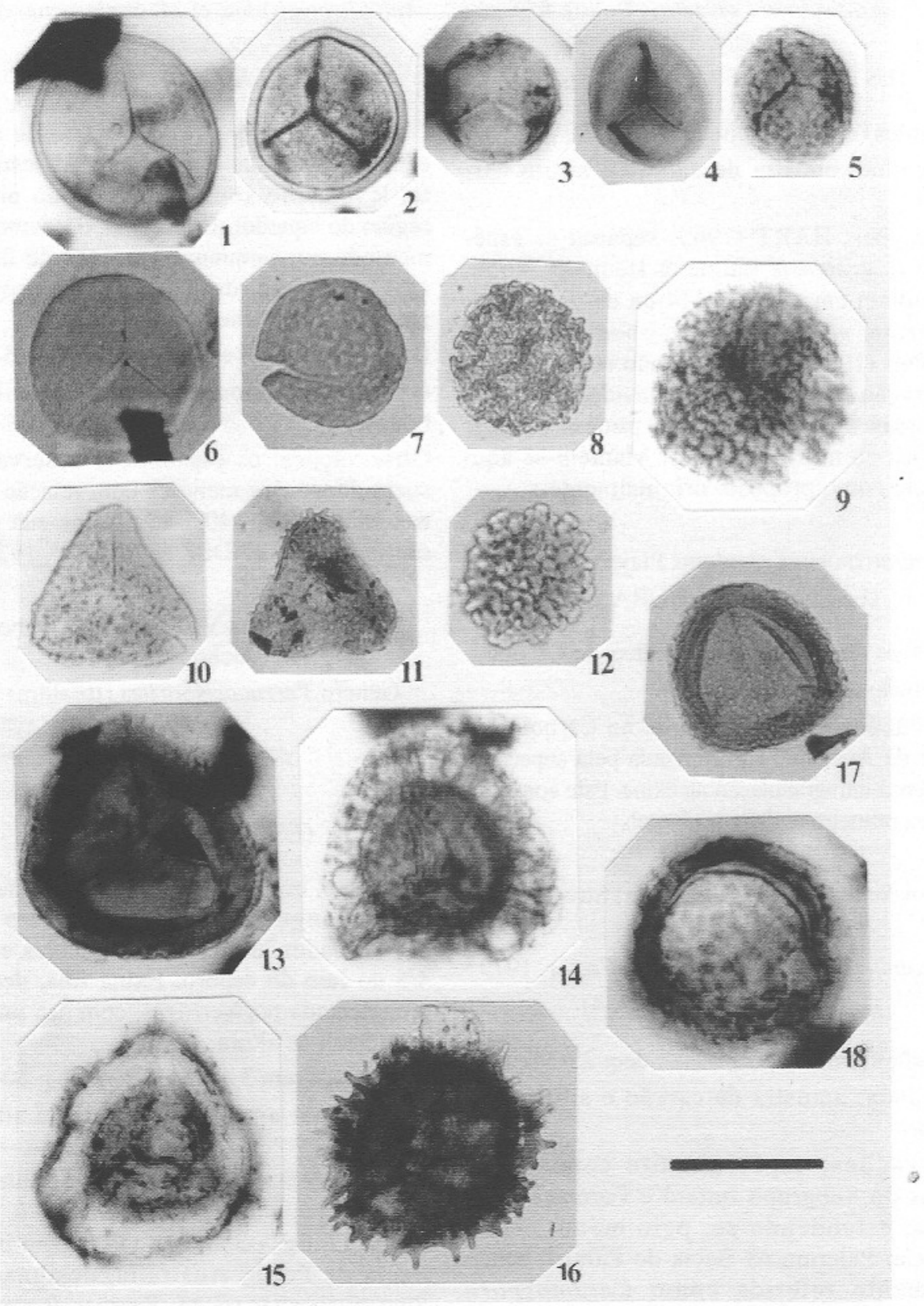

ESTAMPA I - (escala gráfica correspondente a $40 \mu \mathrm{m}$ para as figuras 3, 4, 5, 10, 11 e $65 \mu \mathrm{m}$ para as demais) FIGURA 1 e 6 - Punctatisporites gretensis (Lâmina IG-P-01:131,1/15,1); FIGURA 2 - Punctatisporites resolutus (Lâmina IG-P-02:129,8/23,9); FIGURA 3 - Retusotriletes golatensis (Lâmina IG-P-02); FIGURA 4 Retusotriletes nigritellus (Lâmina IG-P-02); FIGURA 5 - Apiculiretusispora $\mathrm{cf}$. A. coalescens (Lâmina IG-P01); FIGURA 7 - Foveosporites hortonensis (Lâmina IG-P-07:139,0/8,0); FIGURA 8 - Reticulatisporites sp. (Lâmina IG-P-03:139,8/16,8); FIGURA 9 - Verrucosisporites sp. (Lâmina IG-P-02); FIGURA 10 Granulatisporites varigranifer (Lâmina IG-P-02:136,8/23,8); FIGURA 11 - Horriditriletes uruguaiensis (Lâmina IG-P-02:136,9/26,8); FIGURA 12 - Raistrickia rotunda (Lâmina IG-P-07:137,9/10,9); FIGURA 13 Densosporites simplex (Lâmina IG-P-03:135,1/20,5); FIGURA 14 - Vallatisporites arcuatus (Lâmina IG-P02); 15 - Vallatisporites cf. $V$. ciliaris (Lâmina IG-P-07:141,2/17,2); FIGURA 16 - Cristatisporites irradiatus (Lâmina IG-P-06:129,1/8,6); FIGURA 17 - Lundbladispora brasiliensis (Lâmina IG-P-02:133,2/20,8); FIGURA 18 - Cristatisporites menendezii (Lâmina IG-P-02). 
Punctatisporites gretensis Balme \& Hennelly, 1956

(ESTAMPA I, FIGURAS 1 e 6 )

Dimensões (em 5 espécimens): 62,5-80 $\mu \mathrm{m}$. Ocorrência: amostra de carvão e siltito carbonoso.

Observações: HART (1965) separou as espécies de P. gretensis Balme \& Hennelly, 1956, que mediam entre $45(62) 114 \mu \mathrm{m}$ em $P$. gretensis forma minor. Como observado por OTTONE (1991), é difícil e não recomendada a separação desta espécie, considerando suas diferenças de tamanho, que podem refletir variações na mesma espécie. Mantém-se aqui a espécie como proposto originalmente.

\section{Punctatisporites resolutus Playford, 1971} (ESTAMPA I, FIGURA 2)

Dimensões (em 3 espécimens): deq: 65-70 $\mu \mathrm{m}$. Ocorrência: amostra de carvão.

Observações: espécie descrita no Carbonífero Inferior da Austrália, caracterizada pela superfície lisa e densa intragranulação na exina. Este constituise no seu primeiro registro no Brasil.

Gênero Retusotriletes (Naumova) Streel, 1964

Retusotriletes nigritellus (Lüber) Foster, 1979 (ESTAMPA I, FIGURA 4)

Dimensões (em 4 espécimens): deq: 25-34 $\mu \mathrm{m}$. Ocorrência: amostra de carvão e siltito carbonoso.

Observações: espécie muito comum nos estratos do Subgrupo Itararé e Formação Rio Bonito, estendendo-se, pelo menos, até a Formação Palermo na Bacia do Paraná, sendo comumente referida como Calamospora nigritella (Lüber \& Waltz) Hart, 1965.

\section{Retusotriletes golatensis Staplin, 1960} (ESTAMPA I, FIGURA 3)

Dimensões (em 6 espécimens): deq: 24-34 $\mu \mathrm{m}$. Ocorrência: amostra de carvão e siltito carbonoso.

$$
\begin{gathered}
\text { Infraturma APICULATI (Bennié \& Kidston) } \\
\text { Potonié, } 1956
\end{gathered}
$$

Subinfraturma NODATI Dybová \& Jachowicz, 1957

Gênero Apiculiretusispora Streel, 1964
Apiculiretusispora $\mathrm{cf}$. A. coalescens Menéndez \& Azcuy, 1973

\section{(ESTAMPA I, FIGURA 5)}

Descrição: esporo radial trilete de contorno equatorial circular a subcircular. Laesura distinta, levemente sinuosa, chegando próximo à região do equador. Exina fina, densamente ornamentada por pequenas verrugas de $2,5 \mu \mathrm{m}$ de alt. e 1-1,5 $\mu \mathrm{m}$ de base, que se projetam na margem, dando-lhe aspecto irregular.

Dimensões (em 3 espécimens): deq: 25-32 $\mu \mathrm{m}$.

Ocorrência: amostra de carvão e siltito carbonoso.

Observações: os espécimens observados possuem dimensões menores com relação às medidas citadas (deq: 40,5-45 $\mu \mathrm{m}$ ) na sua descrição original (MENÉNDEZ \& AZCUY, 1973).

\section{Subinfraturma VERRUCATI Dybová \& Jachowicz, 1957 \\ Gênero Verrucosisporites (Ibrahim) Smith, 1971}

\section{Verrucosisporites sp. (ESTAMPA I, FIGURA 9)}

Descrição: esporo trilete de contorno subcircular a subtriangular, lados convexos e ângulos arredondados. Marca trilete simples e reta, até $4 / 5$ do raio do esporo. Exina fina, densamente ornamentada por verrugas, com 4-5 $\mu \mathrm{m}$ na base e até $5 \mu \mathrm{m}$ de altura.

Dimensões (em 3 espécimens): deq: 60-75 $\mu \mathrm{m}$. Ocorrência: amostra de carvão e siltito carbonoso.

Observações: as espécies aqui são muito šemelhantes àquela ilustrada por FOSTER (1979: p. 51, pl. 12, fig. 1-3, 5, 6) do Permiano Inferior da Bacia de Perth, Austrália, designada como Bipartitisporites sp. cf. Verrucosisporites trisecatus Balme \& Hennelly, 1956, sobretudo pela densidade dos elementos esculturais (verrugas).

Subinfraturma GRANULATI Dybová \& Jachowicz, 1957

Gênero Granulatisporites (Ibrahim) Potonié \& Kremp, 1954

Granulatisporites varigranifer Azcuy, 1975

(ESTAMPA I, FIGURA 10)

Dimensões (em 3 espécimens): deq: 30-42 $\mu \mathrm{m}$, elementos ornamentais: $0,5-1,0 \mu \mathrm{m}$ de altura.

Ocorrência: amostra de carvão e siltito carbonoso. 
Observações: forma também registrada em Araçoiaba da Serra, Westphaliano da Bacia do Paraná (SOUZA, 1996).

\section{Subinfraturma BACULATI Dybová \& Jachowicz, 1957}

Gênero Horriditriletes Bharadwaj \& Salujha, 1964

\section{Horriditriletes uruguaiensis (Marques-Toigo) Archangelsky \& Gamerro, 1979 \\ (ESTAMPA I, FIGURA 11)}

Dimensões (em 4 espécimens): deq: 34-45 $\mu \mathrm{m}$, báculas: $1,5-2,5 \mu \mathrm{m}$ de base e $5-7,5 \mu \mathrm{m}$ de altura.

Ocorrência: amostra de carvão e siltito carbonoso.

Observações: espécie muito comum nos carvões permianos do Rio Grande do Sul e Santa Catarina. Seus caracteres se encaixam perfeitamente conforme descrição original, sobretudo pela maior incidência da ornamentação nos ângulos.

\section{Gênero Raistrickia (Schopf, Wilson \& Bentall) Potonié \& Kremp, 1954}

\section{Raistrickia rotunda Azcuy, 1975 \\ (ESTAMPA I, FIGURA 12)}

Dimensões (em 7 espécimens): deq: 50-55 $\mu \mathrm{m}$, báculas: $5 \mu \mathrm{m}$ de base e $7,5 \mu \mathrm{m}$ de altura.

Ocorrência: amostra de carvão.

Observações: forma também verificada no carvão de Buri (SOUZA et al., 1990; 1993b) e em Araçoiaba da Serra, Estado de São Paulo (SOUZA, 1996), sendo muito comum em estratos carboníferos da Argentina.

\section{Infraturma MURORNATI Potonié \& Kremp, 1954}

Gênero Foveosporites Balme, 1957

Foveosporites hortonensis Azcuy, 1975 (ESTAMPA I, FIGURA 7)

Dimensões (em 10 espécimens): deq: 50-57 $\mu \mathrm{m}$. Ocorrência: amostra de carvão e siltito carbonoso.

Observações: espécie registrada também no carvão de Buri (SOUZA et al., 1990; 1993b).

Gênero Reticulatisporites (Ibrahim) Potonié \& Kremp, 1954
Reticulatisporites sp.

(ESTAMPA I, FIGURA 8)

Descrição: esporo trilete de contorno equatorial subcircular. Raios da laesura alcançando metade do raio do esporo. Corpo central subcircular, intensamente esculturado com muri, com projeção irregular, de até 6-9 $\mu \mathrm{m}$ de altura.

Ocorrência: amostra de carvão.

Dimensões: deq: 50-58 $\mu \mathrm{m}$.

Suprasubturma LAMINATITRILETES Smith \& Butterworth, 1967

Subturma ZONOLAMINATITRILETES Smith \& Butterworth, 1967

Infraturma CINGULICAVATI Smith \& Butterworth, 1967

Gênero Lundbladispora (Balme) Playford, 1965

Lundbladispora brasiliensis (Marques-Toigo \& Pons) Marques-Toigo \& Picarelli, 1984 (ESTAMPA I, FIGURA 17)

Ocorrência: amostra de carvão.

Dimensões (em 8 espécimens): deq: 57,5-62 $\mu \mathrm{m}$.

\section{Gênero Densosporites (Berry) Butterworth et al., 1964}

Densosporites simplex Staplin \& Jansonius, 1964

(ESTAMPA I, FIGURA 13)

Dimensões (em 4 espécimens): deq: $82-87 \mu \mathrm{m}$, cíngulo: $10-14 \mu \mathrm{m}$.

Ocorrência: amostra de carvão e siltito carbonoso.

Observações: primeiro registro desta espécie no Brasil. Os exemplares observados se encaixam perfeitamente conforme descrição original, sobretudo pelo caráter liso e uniforme do espessamento da exoexina.

Gênero Vallatisporites Hacquebard, 1957

Vallatisporites arcuatus (Marques-Toigo)

Archangelsky \& Gamerro, 1979

(ESTAMPA I, FIGURA 14)

Dimensões (em 11 espécimens): deq: $65-82 \mu \mathrm{m}$ : zona: 14-20 $\mu \mathrm{m}$, corpo central: 35-56 $\mu \mathrm{m}$.

Ocorrência: amostra de carvão e siltito carbonoso. 
Vallatisporites $\mathrm{cf}$ V. ciliaris (Lüber) Sullivan, 1964

(ESTAMPA I, FIGURA 15)

Descrição: esporo radial trilete zonado, de contorno equatorial convexamente triangular. Corpo central liso, bem definido, subtriangular. Raios da laesura nítidos, espessos, levemente sinuosos, alcançando a margem externa da cingulizona. Face proximal finamente infrapunctada. Elementos ornamentais na face distal constituídos de espinhos, mal distribuídos, com projeções nítidas na margem. Cingulizona com engrossamento radial, separando a margem interna.

Dimensões (em 9 espécimens): deq: 60-75 $\mu \mathrm{m}$, zona; $15-20 \mu \mathrm{m}$, corpo central: $35-37,5 \mu \mathrm{m}$.

Ocorrência: amostra de carvão e siltito carbonoso.

Observações: espécime similar à descrita por SOUZA (1996: est. 3, fig. 7) em Araçoiaba da Serra, assinalada na espécie em referência.

\section{Gênero Cristatisporites (Potonié \& Kremp) Butterworth et al., 1964}

\section{Cristatisporites irradiatus (Dias-Fabrício) Picarelli \& Dias-Fabrício, 1990 \\ (ESTAMPA I, FIGURA 16)}

Dimensões (em 7 espécimens): deq: $70-87 \mu \mathrm{m}$, corpo central: $62,5-65 \mu \mathrm{m}$, cingulizona: 7-10 $\mu \mathrm{m}$, elementos ornamentais: até $8 \mu \mathrm{m}$ de altura. Ocorrência: siltito carbonoso.

Observações: espécie comumente registrada no Permiano da Bacia do Paraná, anteriormente referida como C. solaris Dias-Fabrício, 1981.

Cristatisporites menendezii (Menéndez \& Azcuy) Playford emend. Césari, 1986

(ESTAMPA I, FIGURA 18)

Dimensões (em 3 espécimens): deq: 60-84 $\mu \mathrm{m}$, cingulizona: $8-10 \mu \mathrm{m}$.

Ocorrência: amostra de carvão.

Observações: esta espécie também ocorre no material estudado em Araçoiaba da Serra por SOUZA (1996) e se constitui em um bom indicador da idade neocarbonífera para a associação, como ocorre nos estratos argentinos.

\section{Anteturma VARIEGERMINANTES Potonié,} 1970

Turma SACCITES Erdtmann, 1947

Subturma MONOSACCITES (Chitaley)
Potonié \& Kremp, 1954

Infraturma TRILETESACCITES Leschik, 1955

Gênero Cannanoropollis Potonié \& Sah, 1960

Cannanoropollis korbaensis (Bharadwaj \&

Tiwari) Foster, 1975

(ESTAMPA II, FIGURA 2)

Dimensões (em 5 espécimens): deq: 60-95 $\mu \mathrm{m}$, corpo central 55-57 $\mu \mathrm{m}$.

Ocorrência: amostra de carvão e siltito carbonoso.

Cannanoropollis perfectus (Bose \& Maheswari)

Dias-Fabrício, 1981

(ESTAMPA II, FIGURA 1)

Dimensões (em 4 espécimens): deq: 85-110 $\mu \mathrm{m}$, corpo central: $80-85 \mu \mathrm{m}$.

Ocorrência: amostra de carvão e siltito carbonoso.

\section{Gênero Plicatipollenites Lele, 1964}

Plicatipollenites malabarensis (Potonié \& Sah) Foster, 1975

(ESTAMPA II, FIGURA 3)

Dimensões (em 7 espécimens): deq: 106-110 $\mu \mathrm{m}$; corpo central: $73-75 \mu \mathrm{m}$, largura da prega: $10-13 \mu \mathrm{m}$,

Ocorrência: siltito carbonoso.

\section{Infraturma VESICULOMONORADITI Pant, 1954 \\ Gênero Potonieisporites (Bharadwaj) \\ Bharadwaj, 1954}

\section{Potonieisporites methoris (Hart) Foster, 1975 (ESTAMPA II, FIGURA 4)}

Dimensões (em 4 espécimens): eixo longitudinal: $125-145 \mu \mathrm{m}$, transversal: $90 \mu \mathrm{m}$, corpo central no eixo longitudinal: $67,5-70 \mu \mathrm{m}$, no eixo transversal: $70-74 \mu \mathrm{m}$.

Ocorrência: amostra de carvão e siltito carbonoso.

Observações: esta forma é bastante similar àquelas ilustradas por GUTIERREZ (1993: lâm.: 2, fig. 4 e 6), sobretudo pela forma do sistema de pregueamento no corpo central. SOUZA (1996) ilustrou exemplar semelhante a este, $P$. sp. 2 (sua est. 9, fig. 3), porém aquela espécie possui menor desenvolvimento lateral do saco. 

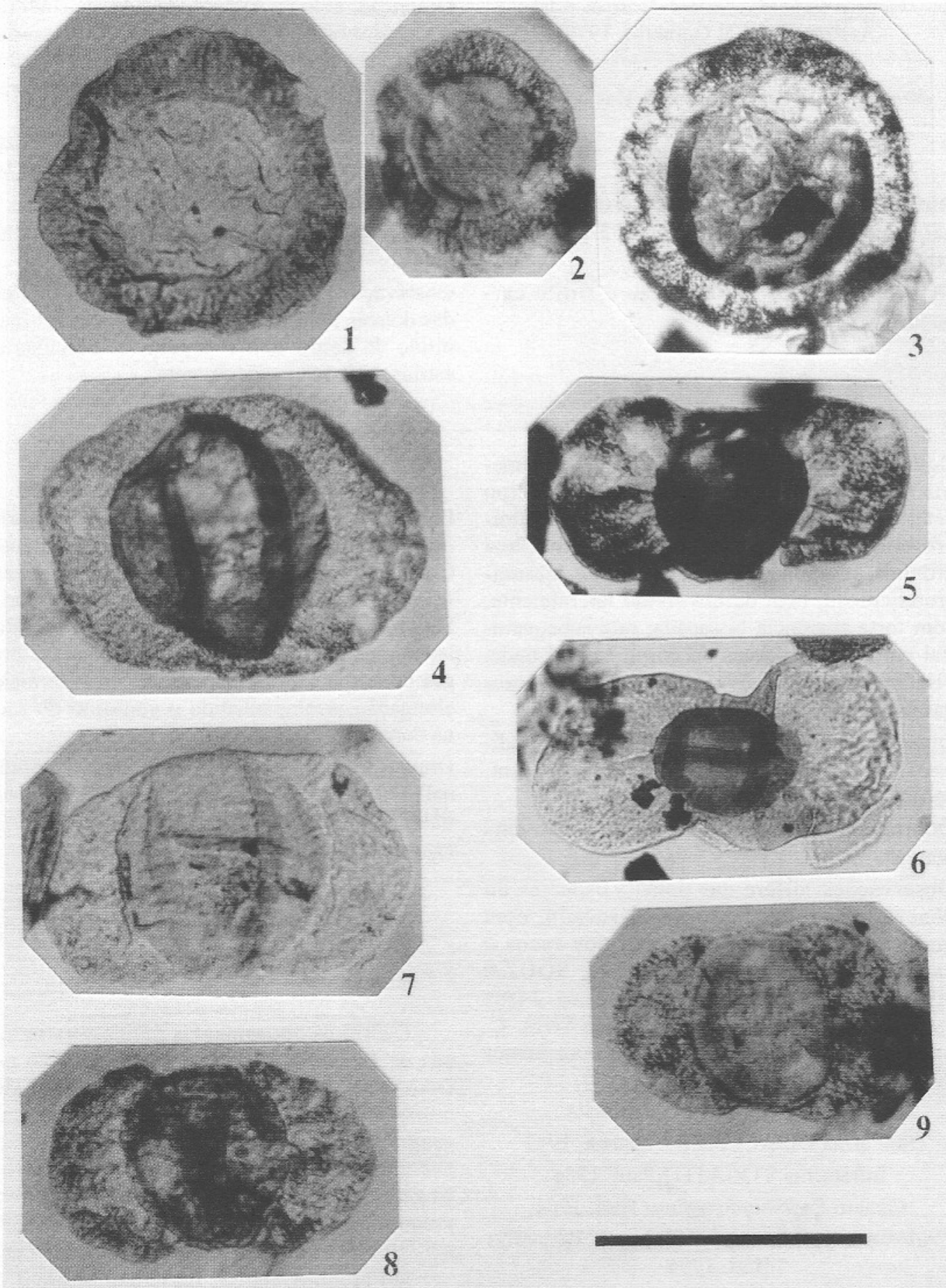

ESTAMPA II - (escala gráfica correspondente a $100 \mu \mathrm{m}$ )

FIGURA 1 - Cannanoropollis perfectus (Lâmina IG-P-06:138,9/8,9); FIGURA 2 - Cannanoropollis korbaensis (Lâmina IG-P-03); FIGURA 3 - Plicatipollenites malabarensis (Lâmina IG-P-07:138,2/10,4); FIGURA 4 Potonieisporites methoris (Lâmina IG-P-07:135,1/13,4); FIGURA 5 - Caheniasaccites ovatus (Lâmina IG-P07:140,8/13,9); FIGURA 6 - Caheniasaccites sp. (Lâmina IG-P-03); FIGURA 7 - Protohaploxypinus amplus (Lâmina IG-P-04:131,8/14,0); FIGURA 8 - cf. Protohaploxypinus sp. (Lâmina IG-P-07:134,1/22,8); FIGURA 9 - Protohaploxypinus sp. (Lâmina IG-P-03:145,1/15,1). 
Gênero Caheniasaccites (Bose \& Kar) Archangelsky \& Gamerro, 1979

\section{Caheniasaccites ovatus (Bose \& Kar) Gutiérrez, 1993}

(ESTAMPA II, FIGURA 5)

Dimensões (em 4 espécimens): eixo longitudinal: $125 \mu \mathrm{m}$, eixo transversal: $55 \mu \mathrm{m}$, corpo central: $55 \mu \mathrm{m}$.

Ocorrência: amostra de carvão e siltito carbonoso.

\section{Caheniasaccites sp. (ESTAMPA II, FIGURA 6)}

Descrição: grão de pólen monossacado de simetria bilateral e contorno diploxilonóide. Corpo central subcircular a ovalado, nítido, intramicroreticulado, com marca monolete na face proximal, até $3 / 4$ do raio deste. Saco intramicroreticulado, bem desenvolvido lateralmente, com forte aparência bissacada; raiz subequatorial em ambas as faces do corpo central onde, distalmente, desenvolve-se um sistema de pregas retangular e nítidas dobras/pregas radiais.

Dimensões (em 3 espécimens): eixo longitudinal: $120-132 \mu \mathrm{m}$, eixo transversal: $70-82 \mu \mathrm{m}$, corpo central: $60-62 \mu \mathrm{m}$.

Ocorrência: amostra de carvão e siltito carbonoso.

Observações: difere das demais espécies do gênero pelo forte contorno bissacado, com maior desenvolvimento lateral dos sacos e expressivas pregas/dobras radiais. SOUZA (1996) descreveu a mesma espécie $C$. sp. 2 (sua est. 10 , fig. 3 e 4 ) em Araçoiaba da Serra. $C$. ovatus e C. densus possuem saco com menor desenvolvimento lateral.

\section{Subturma DISACCITES Cookson, 1947}

Infraturma STRIATITI Pant, 1954

Gênero Protohaploxypinus Hart, 1964

Protohaploxypinus amplus (Balme \& Hennelly) Hart, 1964

(ESTAMPA II, figura 7)

Dimensões (em 4 espécimens): eixo longitudinal: $135 \mu \mathrm{m}$, eixo transversal: $100 \mu \mathrm{m}, 11$ estrias longitudinais.

Ocorrência: amostra de carvão e siltito carbonoso.
Descrição: grão de pólen bissacado, de contorno diploxilonóide, alongado longitudinalmente. Corpo central circular, com 9-10 estrias longitudinais. Sacos intrareticulados, semicirculares, unidos lateralmente.

Dimensões (em 3 espécimens): eixo longitudinal: $85-110 \mu \mathrm{m}$, eixo transversal: $60-78 \mu \mathrm{m}$, corpo central: $55 \mu \mathrm{m}$.

Ocorrência: amostra de carvão e siltito carbonoso.

Observações: os espécimens observados diferem das demais espécies do gênero pelo caráter mais nítido do corpo central e pelas ocorrências de estrias de forma muito discreta.

\section{cf. Protohaploxypinus sp. (ESTAMPA II, figura 8)}

Descrição: grão de pólen bissacado, de contorno diploxilonóide, alongado longitudinalmente. Corpo central circular a ovalado transversalmente, com 9 a 11 estrias longitudinais. Sacos intrareticulados, semicirculares, unidos lateralmente, com raiz distal associada a 2 pregas no corpo central, normais ao eixo de maior elongação, acompanhando o contorno do saco no corpo central.

Dimensões (em 3 espécimens): eixo longitudinal: $105 \mu \mathrm{m}$, eixo transversal: $65 \mu \mathrm{m}$, corpo central: $55 \mu \mathrm{m}$, largura entre sacos: $10 \mu \mathrm{m}$.

Ocorrência: amostra de siltito carbonoso.

\section{DISCUSSÃO DOS RESULTADOS}

\subsection{Correlação bioestratigráfica e idade}

Dentre os zoneamentos palinoestratigráficos da Bacia do Paraná, é o estabelecido por DAEMON \& QUADROS (1970) o de maior utilização, dada sua abrangência estratigráfica e geográfica. Contudo, este tem sido aperfeiçoado nos últimos anos, em razão do detalhamento e da geração de novos dados, resultando em novas propostas de integração, como aquela noticiada por DAEMON \& MARQUES-TOIGO (1991).

No entanto, para a parte setentrional da bacia, ainda há carência de maiores detalhamentos, principalmente dada a existência de novas informações que indicam ter sido nesta parte da bacia o início da sedimentação neopaleozóica da bacia, conforme datações realizadas por vários autores (LIMA et al., 1983; SOUZA et al.,1990, 1993b; DAEMON \& FRANÇA, 1993; SOUZA, 1996).

Como já enfatizado por outros autores (e.g. LIMA \& SUNDARAM, 1982; SOUZA et al., 
1993b), o esquema bioestratigráfico elaborado por DAEMON \& QUADROS (1970) apresenta dificuldades na sua utilização, embora seja o mais completo para a bacia. Uma das razões é que o mesmo foi elaborado somente com base na distribuição dos grãos de pólen. Mesmo em se tratando de um morfogrupo importante do ponto de vista bioestratigráfico, sabe-se que, grosso modo, grãos de pólen monossacados de simetria radial (Cannanoropollis, Plicatipollenites) e bilateral (Caheniasaccites, Potonieisporites) e mesmo alguns estriados (Protohaploxypinus) ocorrem com expressividade no Neocarbonífero em nível mundial, sendo reduzido o número de espéciesguias para algumas idades ou biozonas. Como exemplo pode-se citar que a simples constatação de Plicatipollites malabarensis, Cannanoropollis korbaensis e Protohaploxypinus amplus seria suficiente para enquadrar a associação de Monte Mor nos intervalos G-H.

Geralmente é aceito que as associações contendo altos percentuais de grãos de pólen estriados indicam idades permianas, dada sua ocorrência generalizada neste período. Contudo, seus registros mais primitivos datam do Carbonífero Médio (?) - Superior e, na Bacia do Paraná, têm sido verificado no intervalo $G$ (Protohaploxypinus amplus $=$ forma $\mathrm{P} 486 \mathrm{de}$ DAEMON \& QUADROS, 1970), em sedimentos datados no Carbonífero Superior em Buri (SOUZA et al., 1990, 1993b) e Araçoiaba da Serra (LIMA et al., 1983; SOUZA, 1996). Estes últimos posicionamentos foram realizados com base, principalmente, no registro de alguns esporos inéditos para a bacia, comuns em estratos carboníferos de outras regiões (e.g. espécies dos gêneros Cristatisporites (exAncistrospora), Fovoesporites, Stenozonotriletes, Anapiculatisporites, Raistrickia e Ahrensisporites, entre outras).

Nesse contexto, a associação de Monte Mor pode ser correlacionada com as de Araçoiaba da Serra ou Buri pela ocorrência de esporos comuns, principalmente Cristatisporites menendezii, Foveosporites hortonensis, Granulatisporites varigranifer, Raistrickia rotunda, Vallatisporites ciliaris e Caheniasaccites sp. Por outro lado, as composições percentuais dos morfogrupos verificados são bastante distintas.

Comparando-se as assembléias de Buri e Monte Mor, nota-se que na primeira a participação dos grãos de pólen estriados é quase que nula, representada por um único espécime de Protohaploxypinus sp. Já em Monte Mor, a participação e a variedade deste gênero é notadamente maior, o que pode significar por um lado, controle paleoecológico e por outro, uma ligeira ancestralidade do carvão de Buri. No entanto, verifica-se que em Araçoiaba da Serra a diversificação dos grãos de pólen estriados não é tão inexpressiva, estando ali presentes esporos e grãos de pólen monossacados que lhe asseguram idade westphaliana (e.g. Ahrensisporites cristatus, Cristatisporites spp., Reticulatisporites muricatus, $F$. occultus). Dessa forma, dada a similaridade das associações, é mais prudente indicar a correlação dos níveis de carvão de Monte Mor com aqueles de Buri e, também, com os níveis de Araçoiaba da Serra (superfície e subsuperfície).

Em nível gondvânico, a constatação de Cristatisporites menendezii, Foveosporites hortonensis e Granulatisporites varigranifer sugere correlação com a Palinozona de Ancistrospora (sensu AZCUY \& JELIN, 1980), embora nesta não ocorra grãos de pólen estriados. Por isso, assim como em Buri, é possível que se trate de uma associação transicional entre a Palinozona de Ancistrospora e Palinozona de Potonieisporites da Bacia de Paganzo ou Zona Potonieisporites novicus da Bacia Chaparanense, da Argentina.

A equivalência de idade seria ao Westphaliano, seguramente pré-permiana, pela presença de esporos relativamente primitivos e ausência de grãos de pólens estriados típicos das associações permianas.

Esta análise bioestratigráfica e os resultados alcançados indicam a clara necessidade de um maior refinamento da palinoestratigrafia do Carbonífero na Bacia do Paraná, de modo a deixar também claro as correspondências com as bacias vizinhas do continente sulamericano, onde o volume de dados é relativamente maior.

O posicionamento da assembléia de Monte Mor no Carbonífero Superior corrobora as observações de MILLAN (1975) com base nos dados paleobotânicos. A tafoflora ali presente se constituiria, segundo aquele autor, na mais antiga da Bacia do Paraná, distinta das ocorrentes nos Estados do sul do país, onde são mais freqüentes espécies de Glossopteris e Gangamopteris. Posteriormente, também RÖSLER (1978) indicou a tafoflora de Monte Mor como a associação-tipo da tafoflora mais antiga da bacia. As idades apontadas a partir do inseto ali descrito (MARTINS NETO et al., 1988) e por megásporos (TRINDADE, 1970) corroboram a idade carbonífera superior para Monte Mor.

Do ponto de vista macroflorístico não há possibilidades de diferenciação entre os dois carvões, tendo MILLAN (1987) já citado que Nothorhacopteris sp., único elemento descrito em Buri, poderia representar uma possível 
afinidade com o carvão de Monte Mor. As bases que levaram SOUZA et al. (1990, 1993b) a posicionarem o carvão de Buri como mais antigo que o de Monte Mor foram a maior diversificação e a participação quantitativa dos grãos de pólen estriados neste último, como aqui constatado. Estas evidências não são suficientes para a distinção, tendo em vista tratar-se do mesmo gênero registrado em Buri (Protohaploxypinus). Sua maior diversificação em Monte Mor pode corresponder a diferenças paleoecológicas das associações, não denotando nenhum significado biocronoestratigráfico.

\subsection{Correlação estratigráfica}

SOUZA et al. (1993b) chamam a atenção para o fato da dificuldade dos estudos estratigráficos do Subgrupo Itararé, em função da ausência de níveis-guia regionais e à não utilização (ou emprego) sistemático de análises palinológicas, que se mostram como único método potencial nas correlações bioestratigráficas para a unidade na bacia. Como conseqüência, o entendimento do seu contexto paleogeográfico, bem como as correlações intrabacinais, no âmbito da Bacia do Paraná, são fortemente prejudicadas. Outro fator importante nestas questões, salientado pelos autores, diz respeito à tectônica pós-Itararé, notadamente a mesocenozóica. Como se sabe, a reativação sulatlantiana provocou, entre outras coisas, falhamentos e intromissões de diques e sills de diabásio nos sedimentos paleozóicos da Bacia do Paraná. Como resultado, em alguns casos deslocamentos verticais são tão significativos a ponto de originar falsas interpretações estratigráficas.

Seja por um motivo, seja pelo outro, os depósitos de carvão de Monte Mor têm sido posicionados, até o presente, na parte inferior (MILLAN, 1987) ou média (SAAD, 1977) do Subgrupo Itararé no Estado de São Paulo. No entanto, o resultado palinológico ora obtido mostra que o intervalo analisado corresponde, cronologicamente, à parte basal aflorante desta unidade, na medida em que outros níveis estratigráficos do Itararé têm revelado idades bem mais novas.

Cronoestratigraficamente, os depósitos costeiros de Monte Mor correlacionam-se, grosso modo, aos sedimentos marinhos de Araçoiaba da Serra e aos depósitos de carvão de Buri, e parecem corresponder às primeiras ingressões marinhas detectadas na seqüência permocarbonífera da Bacia do Paraná. Caso isto seja verdade, tudo leva a crer que esta ingressão corresponda ao primeiro ciclo deposicional marinho, registrado em subsuperfície por CAMPANHA et al. (1989), cuja zona de condensação foi chamada de "Folhelho Roncador". Tal seqüência foi registrada no poço 1-RO-1-PR (Roncador n. 1), no intervalo 3.586-3.633 m de profundidade, datado recentemente por R. F. Daemon (1996, comunicação pessoal) como de idade westphaliana.

Muitos dos comentários expostos acima são aqueles mencionados por SOUZA et al. (1993b) na discussão do significado bioestratigráfico do carvão de Buri, se aplicando novamente ao caso de Monte Mor, uma vez que podem ser correlacionados palinologicamente e dizem respeito ao mesmo contexto geológico da bacia.

Conforme salientado por SOUZA et al. (1993b), fora do Brasil, a única possibilidade de correlação é com sedimentos da Bacia de Paganzo (Argentina), cujo conteúdo palinológico mostra-se muito similar. Porém, são necessárias análises mais pormenorizadas para chegar a qualquer correlação litoestratigráfica, principalmente em caráter extra-bacinal.

Com relação às bacias gondvânicas, em especial as australianas, as correspondências só podem ser estabelecidas com base em algumas características gerais das assembléias (percentuais de morfogrupos), uma vez que são poucos os táxons comuns.

\subsection{Informações paleoambientais}

O conjunto microflorístico ora identificado, com predomínio de elementos tipicamente continentais, constituído principalmente por representantes de licófitas (Densosporites, Ancistrospora, Vallatisporites, Cristatisporites, Lundbladispora) e filicófitas (Punctatisporites, Raistrickia, Granulatisporites, Horriditriletes), é indicativo de terras baixas e úmidas, provavelmente próximo a algum corpo d'água. A relação percentual dos morfogrupos observados pode ser verificada nas FIGURAS 3 e 4.

Como ocorre na maioria das assembléias palinológicas do Neocarbonífero, em nível gondvânico ou extra-gondvânico, as assembléias de Monte Mor se caracterizam pelo predomínio de esporos e pela participação freqüente de grãos de pólen de simetria radial. Grãos de pólen estriados, embora com participação reduzida, são representados por espécies do gênero Protohaploxypinus, muito comum nas assembléias neocarboníferas do Gondvana. 
O carvão de Monte Mor, bem como o de Buri, pelas suas composições palinológicas seriam equivalentes às associações LundbladisporaPunctatisporites-Portalites e VallatisporitesCristatisporites, conforme classificação de MARQUES TOIGO \& CORRÊA DA SILVA (1984), o que reforça a idéia de um paleoambiente de terras baixas.

Siltito carbonoso

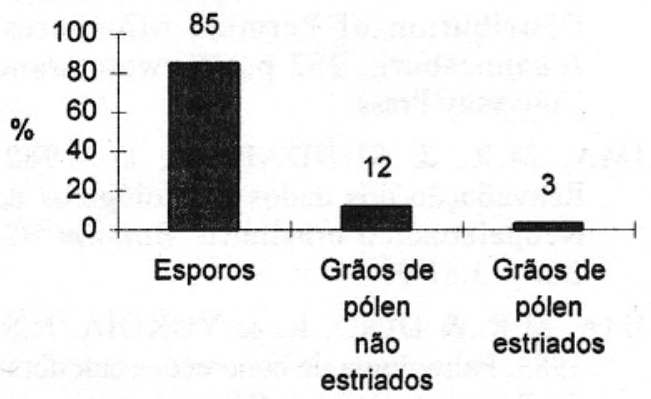

FIGURA 3 - Freqüência dos morfogrupos palinológicos na amostra de siltito carbonoso.

\section{Carvão}

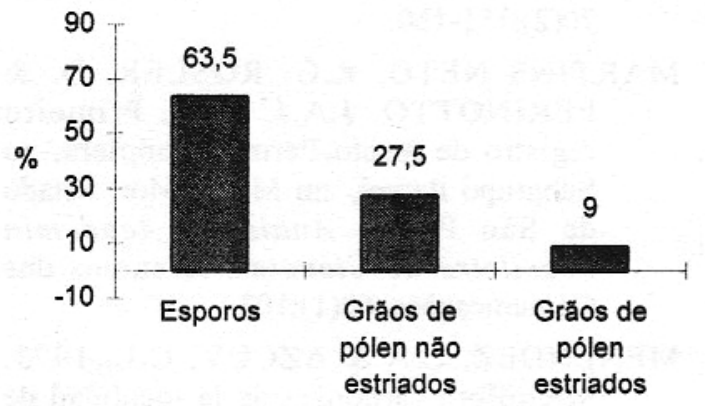

FIGURA 4 - Freqüência dos morfogrupos palinológicos na amostra de carvão.

A origem semi-autóctone para o carvão é indicada pelos megásporos, macrofósseis vegetais da capa do carvão em boas condições de fossilização e mesmo pelo ritmismo observado nas camadas de folhelho (MILLAN, 1975).

\section{PRINCIPAIS CONCLUSÕES}

A análise da composição microflorística do carvão de Monte Mor e a comparação dos resultados com outros dados já divulgados permitem, em termos gerais, enumerar as seguintes conclusões:

1. os níveis portadores de carvão da região de Monte Mor registram uma palinoflora diversificada, cujo domínio é de esporos triletes (lisos, apiculados, zonados e cingulizonados), com participação subordinada de grãos de pólen (monossacados, bissacados), estriados ou não;

2. na Bacia do Paraná, as assembléias palinológicas com características e elementos comuns são as descritas em Araçoiaba da Serra e em Buri. Estas devem corresponder a uma palinozona carbonífera, equivalente à "Pré-G", informalmente designada por LIMA et al. (1983), com correspondência bioestratigráfica, em nível gondvânico, mais claramente observada na Argentina (entre as palinozonas de Ancistrospora e Potonieisporites);

3. apesar da composição palinológica muito semelhante à da Formação Malazán da Bacia de Paganzo (Argentina), é prematura ainda qualquer correlação litoestratigráfica desta com os níveis de carvão de Monte Mor ou com as outras associações brasileiras (Buri e Araçoiaba da Serra);

4. é sugerida uma idade westphaliana, neocarbonífera, à associação de Monte Mor, tal como fôra para Buri e Araçoiaba da Serra;

5. o domínio de representantes da paleoflora continental, aliado às interpretações de caráter litofaciológico, conduzem à reconstituição de um cenário paleogeográfico caracterizado por planície deltaica de um sistema deltaico aos níveis portadores do carvão de Monte Mor.

\section{AGRADECIMENTOS}

Os autores expressam sinceros agradecimentos aos profs. Dr. Paulo Roberto dos Santos e José Henrique Millan pela cessão das amostras, à Dra. Gianna Maria Garda (IG/SMA) pela revisão do Abstract, bem como aos revisores do trabalho pelas sugestões apresentadas. 


\section{REFERÊNCIAS BIBLIOGRÁFICAS}

ARCHANGELSKY, S. \& CUNEO, R. 1991. The Neopaleozoic floristic succession from Northwestern argentina. A new perspective. In: ULBRICH, H. \& ROCHACAMPOS, A.C. (Editors) Gondwana Seven. SEVENTH INTERNATIONAL GONDWANA SYMPOSIUM, São Paulo, 1988. Proceedings... p. 469-481.

AZCUY, C.L. \& JELIN, R. 1980. Las palinozonas e o limite Carbono-Permico en la Cuenca Paganzo. In: CONGRESO ARGENTINO DE PALEONTOLOGIA Y BIOESTRATIGRAFIA, 2., CONGRESO LATINOAMERICANO DE PALEONTOLOGIA, 1., 1980, Buenos Aires. Actas. 4:51-67.

BARBOSA, O. 1958. On the age of the Lower Gondwana floras in Brazil and abroad. In: CONG. GEOL. INTERNAT., 20., 1956, México. Com. de Gonduana, p. 205-236.

BARBOSA, O. \& ALMEIDA, F.F.M. 1949. A Série Tubarão na bacia do rio Tietê, Estado de São Paulo. Notas Preliminares e Estudos, Div. Geol. Miner., DNPM, 48:1-16.

CAMPANHA, V.A., SAAD, A.R., GAMA JUNIOR, E. \& PULENGUI FILHO, P. 1989. A caracterização marinha do Grupo Itararé (Permocarbonífero), Bacia do Paraná. In: SIMPÓSIO DE GEOLOGIA DO SUDESTE, 1., 1989, Rio de Janeiro. Boletim de Resumos. SBG, p. 5.

DAEMON, R.F. 1974. Integração dos resultados palinológicos de fauna e flora das camadas fossilíferas da Bacia do Paraná - Implicações estratigráficas e paleogeográficas. Rev. Unimar, 1:25-40.

DAEMON, R.F. \& QUADROS, L.P. 1970. Bioestratigrafia do Neopaleozóico da Bacia do Paraná. In: CONGRESSO BRASILEIRO DE GEOLOGIA, 24., 1970, Brasília. Anais... SBG, p. 359-412.

DAEMON, R.F. \& MARQUES TOIGO, M. 1991. An integrated biostratigraphical column for the Paraná Basin, Brazil. In: INTERNATIONAL CONGRESS OF CARBONIFEROUS AND PERMIAN STRATIGRAPHY AND GEOLOGY 12., 1991, Buenos Aires. Abstracts. p. 25-26.

DAEMON, R.F. \& FRANÇA, A.B. 1993. Sedimentos do Westfaliano (Carbonífero Médio) na Formação Lagoa Azul, Grupo Itararé. In: CONGRESSO SOBRE CRONOESTRATIGRAFIA DA BACIA
DO PARANÁ, 1., 1993, Rio Claro. Resumos. UNESP, p. 36.

FOSTER, C.B. 1979. Permian plant microfossils from the Blair Athol Coal Measures, Baralaba Coal Measures, and Basal Rewan Formation of Queensland. Geological Survey of Queensland, Publ. 372, Paleontological Paper 45:1-154.

GUTIERREZ, P.R. 1993. Palinologia de la Formacion Agua Colorada (Carbonífero Superior), Sierra de Famatina, Provincia de La Rioja, Argentina. I. Granos de polen. Ameghiniana, 30(2):163-212.

HART, G.F. 1965. The Systematic and Distribution of Permian Miospores. Johannesburg, 252 p., Witwatersrand University Press.

LIMA, M.R. \& SUNDARAM, D. 1982. Reavaliação dos dados palinológicos do Neopaleozóico brasileiro. Boletim IGUSP, 13:81-99.

LIMA, M.R. \& DINO, R. \& YOKOIA, N.S. 1983. Palinologia de concreções calcíferas do Subgrupo Itararé (Neopaleozóico da Bacia do Paraná) na região de Araçoiaba da Serra, Estado de São Paulo. Anais da Academia Brasileira de Ciências, 55(2): 195-208.

MARQUES TOIGO, M. \& CORREAA DA SILVA, Z. 1984. On the origin of Gondwanic South Brazilian coal measures. Comun. Serv. Geol. Portugal, 70(2):151-160.

MARTINS NETO, R.G. RÖSLER, O. \& PERINOTTO, J.A.J. 1988. Primeiro registro de inseto Permotrichoptera, no Subgrupo Itararé, em Monte Mor, Estado de São Paulo. Anais da Academia Brasileira de Ciências, Resumos das Comunicações, 60(1):103.

MENÉNDEZ, C.A \& AZCUY, C.L. 1973. Microflora carbonica de la localidad de Paganzo, Provincia de La Rioja. Parte III. Ameghiniana, X (1): 51-71.

MILLAN, J.H. 1972. Macroflórula carbonifera de Monte Mor, Estado de São Paulo. São Paulo, 165 p. (Tese de Doutorado, Instituto de Geociências da USP). Inédita.

MILLAN, J.H. 1975. Tafoflórula Monte Mor do Estado de São Paulo: seus elementos e seu significado no Gondwana Inferior do Brasil. Revista Brasileira de Geociências, 5:1-14. 
MILLAN, J.H. 1981a. Sobre a presença das Sphenophyllales no Eogondwana de Monte Mor, Subgrupo Itararé do Estado de São Paulo, Brasil. In: CONGRESSO LATINOAMERICANO DE PALEOBOTÂNICA E PALINOLOGIA, II., 1981, Porto Alegre, Actas. v. 1:113-125.

MILLAN, J.H. 1981b. Sobre a presença de folhas cf. Ginkgophylum no Eogondwana de Monte Mor, Subgrupo Itararé do Estado de São Paulo. Bol. Museu Nac., N. S. Geol., 39:1-12.

MILLAN, J.H. 1987. Os pisos florísticos do carvão do Subgrupo Itararé do Estado de São Paulo e suas implicações. In: CONGRESSO BRASILEIRO DE PALEONTOLOGIA, X., 1987, Rio de Janeiro. Anais... SBP, p. 831-857.

OTTONE, E.G. 1991. Palynologie du Carbonifère Supérieur de la Coupe de Mina Esperanza, Bassin Paganzo, Argentina. Revue de Micropaléontologie, 34(2):118-135.

PETRI, S. \& SOUZA, P.A. 1993. Síntese e novas concepções sobre a bioestratigrafia do Subgrupo Itararé. Revista do Instituto Geológico, 14(2):7-20.

POTONIÉ, R. 1970. Synopsis der Gattungen der Sporae Dispersae. V Teil: Nachträge zur allen Gruppen (Turmae). Geologischen Jahrbuch, 87:1-172.

RÖSLER, O. 1978. The Brazilian Eogondwanic Floral Succession. Bol. IG/USP, Advances in palaeobotany and Allied Sciences in Brazil, 9:85-91.

SAAD, A.R. 1977. Estratigrafia do Subgrupo Itararé no centro e sul do Estado de São Paulo. São Paulo, 107 p. (Dissertação de Mestrado, Instituto de Geociências da USP). Inédita.

SANTOS, P.R. 1979. Distribuição estratigráfica, características e fácies de diamictitos e rochas associadas do
Subgrupo Itararé no centro e sul do Estado de São Paulo. São Paulo, 135 p. (Dissertação de Mestrado, Instituto de Geociências da USP). Inédita.

SANTOS, P.R. 1987. Fácies e evolução paleogeográfica do Subgrupo Itararé/Grupo Aquidauana (Neopaleozóico) na Bacia do Paraná, Brasil. São Paulo, 128 p., v. I (Tese de Doutorado, Instituto de Geociências da USP). Inédita.

SOUZA, P.A. 1996. Palinologia $e$ bioestratigrafia do Subgrupo Itararé em Araçoiaba da Serra (Westphaliano, Bacia do Paraná), Estado de São Paulo, Brasil. São Paulo, 192 p., 12 est. (Dissertação de Mestrado, Instituto de Geociências da USP). Inédita.

SOUZA, P.A., LIMA, M.R. \& SAAD, M.R. 1990. Palinologia dos carvões paleozóicos do Estado de São Paulo. I-O Carvão de Buri. Paleobotânica Latinoamericana (Circular Informativa da ALPP), 9(1):55.

SOUZA, P.A., LIMA, M.R. \& SAAD, M.R. 1993a. Palinologia dos carvões paleozóicos do Estado de São Paulo. II-O Carvão de Monte Mor. Anais da Academia Brasileira de Ciências, Resumo das Comunicações, 65(3):327.

SOUZA, P.A., LIMA, M.R. \& SAAD, M.R. 1993b. Palinologia dos carvões paleozóicos do Estado de São Paulo. I-O Carvão de Buri. Revista do Instituto Geológico, 14(1):5-20.

SOUZA FILHO, E.E. 1986. Mapeamento faciológico do Subgrupo Itararé na Quadrícula de Campinas (SP). São-Paulo, 121 p. (Dissertação de Mestrado, Instituto de Geociências da USP). Inédita.

TRINDADE, N.M. 1970. Megásporos carboníferos de Monte Mor, Estado de São Paulo. Anais da Academia Brasileira de Ciências, 42(3):459-470. 